\title{
Update from RCP Quality Improvement: Improvement opportunities and challenges in going digital and distant
}

\section{DOI: 10.7861/fhj.QI-7-3}

Quality improvement (QI) is fundamentally about people and processes of care. Bringing together staff, patients and families in the workplace and our practice is core to all improvement work. This has been seriously challenging during the novel coronavirus pandemic, but it has been a stimulus to reassess how we do this. Improvement support teams were often redeployed, and huge amounts of process change happened at a pace not usually experienced. The effects of these changes were not commonly measured, so was there improvement?

However, digital platforms have been embraced to bring people together, both for meetings and for care delivery. A common perception is that this has brought people together more easily but evaluation is needed, particularly because some of our most vulnerable patients and staff may be 'digitally excluded'. How we do QI has changed because of these factors, and learning about how change has happened has been explored. ${ }^{2}$

Examining the benefits and limitations of using digital platforms for QI work has also helped us examine our traditional approaches. ${ }^{3}$ Within Royal College of Physicians (RCP) QI and Patient Safety, our activities have significantly changed during this pandemic, much was based on bringing people together in person to learn from and with each other. We have questioned and clarified when it is important for people to be in the same physical space, and the limitations that it might bring for accessibility and the unhelpful hierarchies. People build relationships by being in the same physical space, this fosters trust and then openness, which is important when sharing what works and what doesn't work in clinical care and improvement practice. It is probably easier to adapt the way we interact or create learning when we are in a physical space together, and multiple interactions can happen informally. Not forgetting how we use the sticky note, that often used QI tool. Observing and interacting with staff and patients in the clinical setting brings vital insights to improving care.

With digital delivery, information can be available for access when it's convenient or on multiple occasions, rather than just in a timed session. Other aspects should be delivered synchronously for all participants when real-time interactions are needed, or at critical points in an improvement programme. Digital delivery isn't necessarily limited by room size, meaning more people can be involved, although sense of community is different with mass participation. Digital platforms and how we use them are developing to enable improvement work. When are small or large groups beneficial? How long should sessions be? How do we best use interactive white boards, discussion mechanisms, and even the electronic shared sticky note and flipchart? Can we work at a different pace when using these approaches? Some have suggested that that power hierarchies are diminished through digital media and that should help inclusiveness and learning. Within RCP QI, we had to pause a planned comprehensive programme of breakthrough collaboratives, the QI faculty development programme and the development of additional programmes to build improvement expertise. We have moved to monthly network 'calls' for those working in improvement, patient safety and patient partnerships, covering areas around COVID-19 recovery, learning from the initial COVID-19 response, digital delivery (that has informed this article), and human elements of change. If you are interested in joining these, please contact RCPQI@rcplondon.ac.uk. The chief registrar programme has been delivered through digital platforms in collaboration with colleagues in education. Improvement collaboratives are being remodelled in line with our learning and incorporating improvement coaching. The first will be for modern ward rounds, improving multidisciplinary inpatient care. Contact wardrounds@rcplondon.ac.uk if you would like to participate. We are also working across departments to develop a digital platform Medical care: Driving change that will share innovative, best practice and improvement materials.

Dr John Dean

Clinical director of quality improvement and patient safety, Royal College of Physicians

\section{References}

1 Ignatowicz $\mathrm{A}$, Atherton $\mathrm{H}$, Bernstein $\mathrm{C}$ ] et al. Internet videoconferencing for patient-clinician consultations in long-term conditions: A review of reviews and applications in line with guidelines and recommendations. Digital Health 2019;5:1-27.

2 Lewis R, Pereira P, Thorlby R, Warburton W. Understanding and sustaining the health care service shifts accelerated by COVID-19. The Health Foundation, 2020. www.health.org.uk/publications/longreads/understanding-and-sustaining-the-health-care-service-shiftsaccelerated-by-COVID-19 [Accessed 17 September 2020].

3 NHS Horizons. \#VirtualCollaborate: Health and care improvement in a virtual world. NHS. http://horizonsnhs.com/communities/ virtualcollaborate [Accessed 08 September 2020]. 